\title{
Predicting prognosis using molecular profiling in estrogen receptor-positive breast cancer treated with tamoxifen
} Sherene Loi $^{\dagger 1,2}$, Benjamin Haibe-Kains ${ }^{\dagger 1,3}$, Christine Desmedt ${ }^{1}$, Pratyaksha Wirapati ${ }^{4}$, Françoise Lallemand ${ }^{1}$, Andrew M Tutt ${ }^{5}$, Cheryl Gillet ${ }^{5}$, Paul Ellis ${ }^{5}$, Kenneth Ryder ${ }^{5}$, James F Reid ${ }^{6,8}$, Maria G Daidone ${ }^{8}$, Marco A Pierotti6,8, Els MJJ Berns7, Maurice PHM Jansen7, John A Foekens ${ }^{7}$, Mauro Delorenzi ${ }^{4}$, Gianluca Bontempi ${ }^{3}$, Martine J Piccart ${ }^{1}$ and Christos Sotiriou*1

Address: ${ }^{1}$ Functional Genomics Unit, Jules Bordet Institute, Brussels, Belgium, ${ }^{2}$ Peter MacCallum Cancer Center, East Melbourne, Victoria, Australia, ${ }^{3}$ Machine Learning Group, Université Libre de Bruxelles, Brussels, Belgium, ${ }^{4}$ NCCR Molecular Oncology, Swiss Institute of Cancer Research and Swiss Institute of Bioinformatics, Epalinges, Switzerland, ${ }^{5}$ Guys Hospital, London, UK, ${ }^{6}$ Molecular Cancer Genetics, Fondazione Istituto FIRC di Oncologia Molecolare (IFOM), Milan, Italy, ${ }^{7}$ Erasmus MC-Daniel-JNI, Rotterdam, The Netherlands and ${ }^{8}$ Department of Experimental Oncology, Fondazione IRCCS Istituto Nazionale dei Tumori, Milan, Italy

Email: Sherene Loi - sherene.loi@petermac.org; Benjamin Haibe-Kains - bhaibeka@ulb.ac.be; Christine Desmedt - christine.desmedt@bordet.be; Pratyaksha Wirapati - Pratyaksha.Wirapati@unil.ch; Françoise Lallemand - francoise.lallemand@bordet.be; Andrew M Tutt - andrew.tutt@icr.org.uk; Cheryl Gillet - cheryl.gillet@cancer.org.uk; Paul Ellis - paul.ellis@gstt.sthames.nhs.uk; Kenneth Ryder - kenneth.ryder@cancer.org.uk; James F Reid - james.reid@ifom-ieo-campus.it; Maria G Daidone - mariagrazia.daidone@istitutotumori.mi.it; Marco A Pierotti - marco.pierotti@istitutotumori.mi.it; Els MJJ Berns - p.berns@erasmus.nl; Maurice PHM Jansen - m.p.h.m.jansen@erasmusmc.nl; John A Foekens - j.foekens@erasmusmc.nl; Mauro Delorenzi - Mauro.Delorenzi@isb-sib.ch; Gianluca Bontempi - gbonte@ulb.ac.be; Martine J Piccart - martine.piccart@bordet.be; Christos Sotiriou* - christos.sotiriou@bordet.be

* Corresponding author †Equal contributors

\section{Published: 22 May 2008}

BMC Genomics 2008, 9:239 doi:| 0.| | 86/|47|-2|64-9-239
Received: 28 January 2008

Accepted: 22 May 2008

This article is available from: http://www.biomedcentral.com/I47I-2164/9/239

(C) 2008 Loi et al; licensee BioMed Central Ltd.

This is an Open Access article distributed under the terms of the Creative Commons Attribution License (http://creativecommons.org/licenses/by/2.0), which permits unrestricted use, distribution, and reproduction in any medium, provided the original work is properly cited.

\begin{abstract}
Background: Estrogen receptor positive (ER+) breast cancers $(B C)$ are heterogeneous with regard to their clinical behavior and response to therapies. The ER is currently the best predictor of response to the anti-estrogen agent tamoxifen, yet up to $30-40 \%$ of $E R+B C$ will relapse despite tamoxifen treatment. New prognostic biomarkers and further biological understanding of tamoxifen resistance are required. We used gene expression profiling to develop an outcomebased predictor using a training set of $255 \mathrm{ER}+\mathrm{BC}$ samples from women treated with adjuvant tamoxifen monotherapy. We used clusters of highly correlated genes to develop our predictor to facilitate both signature stability and biological interpretation. Independent validation was performed using 362 tamoxifen-treated ER+ BC samples obtained from multiple institutions and treated with tamoxifen only in the adjuvant and metastatic settings.
\end{abstract}

Results: We developed a gene classifier consisting of $|8|$ genes belonging to $\mid 3$ biological clusters. In the independent set of adjuvantly-treated samples, it was able to define two distinct prognostic groups (HR 2.0I 95\%Cl: 1.29-3.13; $p=0.002$ ). Six of the 13 gene clusters represented pathways involved in cell cycle and proliferation. In $1 / 2$ metastatic breast cancer patients treated with 
tamoxifen, one of the classifier components suggesting a cellular inflammatory mechanism was significantly predictive of response.

Conclusion: We have developed a gene classifier that can predict clinical outcome in tamoxifentreated ER+ BC patients. Whilst our study emphasizes the important role of proliferation genes in prognosis, our approach proposes other genes and pathways that may elucidate further mechanisms that influence clinical outcome and prediction of response to tamoxifen.

\section{Background}

Breast cancers are biologically heterogeneous with regards to their clinical behavior and response to therapies. However, treatment-decision making for women diagnosed with breast cancer is still reliant on classical histopathological appearance and immunohistochemical markers that give little insight into tumor biology and potential response to treatment. There are a few biomarkers routinely used that can predict response to commonly prescribed therapies. The presence of estrogen receptors is the best indicator of response to anti-estrogen agents such as tamoxifen. However, $30-40 \%$ of women with estrogen receptor-positive breast cancer $(\mathrm{ER}+\mathrm{BC})$ will develop distant metastases and die despite tamoxifen treatment. The underlying biological mechanisms of resistance to tamoxifen are incompletely understood.

Gene expression profiling of tumors appears to be a promising new strategy for predicting clinical outcome in breast cancer patients. Recent studies have proposed that the heterogeneity of clinical response can be correlated with different molecular "portraits" [1,2]. Gene signatures have been developed that can distinguish subgroups of patients with different prognoses or response to chemotoxic and antiestrogen agents. However, issues have emerged since these initial studies relating to design and validation of gene classifiers [3], particularly the small numbers of patient samples used to derive the classifier and the little overlap in these gene signatures. Furthermore, it has been shown that membership in a prognostic gene list is not necessarily indicative of a gene's importance in cancer pathology [4]. Extracting biological meaning from whole genome molecular profiling remains a significant challenge.

We have recently shown that in $\mathrm{ER}+\mathrm{BC}$, its proliferative status is the most important predictor of prognosis in these women [5]: highly proliferative tumors have a worst clinical outcome, either with or without systemic treatment. However, proliferation is a downstream consequence and the understanding of the upstream activators is essential for advancing biological knowledge and development of targeted approaches that may be tested in the clinical setting, potentially in combination with antiestrogen agents. In this study, we hypothesized that developing a gene classifier using clusters of correlated genes as single variables may allow for both prediction of clinical outcome in tamoxifen-treated patients and facilitate new biological understanding of resistance mechanisms as these clusters could represent biological networks or pathways. Furthermore, we assessed the performance of our classifier on several independent data sets of tamoxifentreated samples, both in the adjuvant as well as advanced setting. These were obtained from a number of institutions and samples had been hybridized on varying microarray platforms.

\section{Methods \\ Tamoxifen-treated dataset used in development of the classifier}

The dataset used for training the classifier consisted of 255 early-stage (stage I, II) BC samples, diagnosed between 1980 and 1995, all of whom had received tamoxifen only as their adjuvant treatment (hereby referred to as the "tamoxifen-treated dataset"). The demographics can be found in [Additional file 1], and data processing methods are described in Loi et al. [6] as a large proportion of this dataset has been previously used in another research study. The raw data for the tamoxifen-treated dataset are available at the GEO database (accession number GSE6532). This dataset contained samples from the John Radcliffe Hospital (OXFT), Oxford, United Kingdom, Guys Hospital (GUYT), London, United Kingdom and Uppsala University Hospital (KIT), Uppsala, Sweden. All samples had been hybridized using Affymetrix U133 Genechips $^{\text {TM }}$ (HG-U133A, B for OXFT and KIT, and PLUS2 for GUYT). All samples were required to be estrogen (ER) and/or progesterone receptor (PR) positive by ligandbinding assay and had been prescribed tamoxifen monotherapy for 5 years post diagnosis as adjuvant therapy. The cut-off value for classification of patients as positive or negative for ER and PR was $10 \mathrm{fmol}$ per mg protein. The primary endpoint used for generating the classifier was the first distant metastatic event (distant metastasis free survival, DMFS), as survival can be confounded by local recurrence and treatments given at relapse. Each hospital's institutional ethics board approved the use of the tissue material for the purposes of this research study.

\section{Statistical methods}

Figure $1 \mathrm{a}$ and $1 \mathrm{~b}$ summarize the method used to develop the gene classifier. 
A

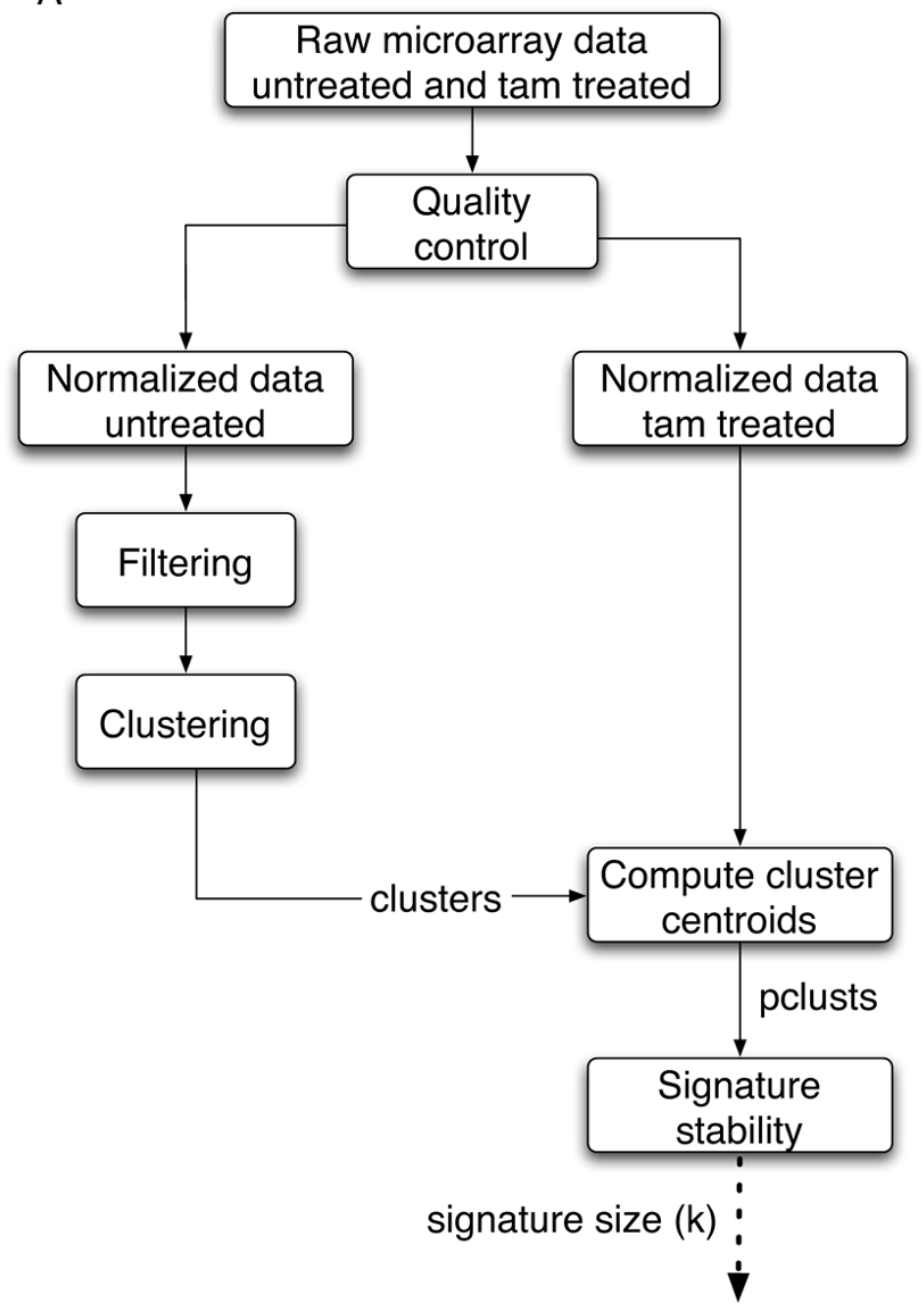

B

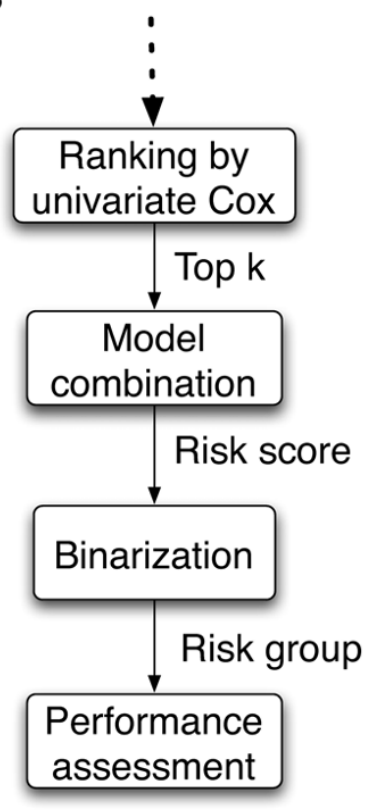

\section{Figure I}

Overview of the analysis design. (a) First part of the analysis including quality controls, normalization, preliminary clustering performed on the untreated dataset, computation of the cluster centroids on the tamoxifen treated dataset, and estimation of signature stability with regards to signature size, using cross-validation. (b) Second part of the analysis including the classifier development, performance assessment by cross-validation and performance assessment on independent validation data sets.

\section{Preliminary clustering}

We used a clustering method in order to identify clusters of highly correlated genes, prior to feature selection and model building as we hypothesized that this would to reduce the number of variables, increase signature stability, allow platform independency and to preserve biological interpretation [8]. Preliminary clustering was performed on separate dataset consisting of 137 samples from untreated women with early stage breast cancer (data available from GEO database, accession number GSE6532). These samples were not used in the signature development to avoid any possible overfitting when performing the cluster identification. Control probe sets and those absent in at least $95 \%$ of the samples were removed.
The data set was then filtered based on overall variance with the top $20 \%$ of probe sets selected for further clustering. Hierarchical clustering with Pearson correlation similarity metric and complete linkage was used. The generated dendrogram was then cut at a height of 0.5 . Clusters were discarded if there were less than 5 known genes (as per Unigene) per cluster. After this procedure, a total of 110 clusters were obtained for signature development [Additional file 2a]. Of note, these clusters were able to be reproduced in the tamoxifen-treated population (data not shown). The cluster centroid, i.e. the average expression level of all the probes per cluster, was then obtained for each cluster in the tamoxifen-treated dataset. 
Each cluster was subsequently treated as a single variable called a "probe cluster" (pclust).

\section{Feature selection}

Although the preliminary clustering significantly reduced the dimensionality of the data, the number of features remained too large to efficiently build the classifier. The selection of the most relevant pclusts was performed using a ranking based on the likelihood ratio statistic of univariate Cox model. The Cox model specifies the hazard of a patient $i$ as

$$
\lambda_{i}(t)=\lambda_{0}(t) \exp \left(\beta p c l u s t_{j}\right),
$$

where $\lambda_{0}(t)$ is the baseline hazard assumed to be equal for all patients (proportional hazards), and pclust $t_{j}$ is the $j$ th pclust of patient $i$. The likelihood ratio statistic is twice the difference in the log partial likelihood between the null model ( $\beta$ is equal to 0 ) and the model with estimate of $\beta$. The only parameter of this feature selection is the signature size, i.e. the number $k$ of pclusts that will be used to build the classification model.

\section{Signature size and stability}

Signature size was set in order to maximize a stability criterion using a multiple 10-fold cross-validation algorithm (M10FOLDCV) on the tamoxifen-treated dataset after randomization of the order of patients in the data set. For each signature size, a criterion designed to estimate the stability of a signature was computed, as recently introduced by Davis and colleagues [9]. For a given signature size $k$, let $P$ be the list of all pclusts. Let $f r e q(p)$ be the number of sampling steps in which the probe cluster $p \in$ $P$ has been selected out of a total of $m$ sampling steps. The set $P$ is sorted by frequency into the set $p_{(1)}, p_{(2)}, \ldots, p_{(n)}$ such that $f r e q\left(p_{(i)}\right) \geq \operatorname{freq}\left(p_{(j)}\right)$ if $i<j$ where $i, j \in\{1,2, \ldots, n\}$. The stability statistic for a signature size $k$ is defined as

$$
\operatorname{Stab}(k)=\frac{\sum_{i=1}^{k} \operatorname{freq}\left(p_{i}\right)}{k m}
$$

The Stab statistic is equal to 1 if the same signature is always selected over M10FOLDCV given a signature size and $\frac{1}{m}$ if there is no overlap. It must be noted that Stab statistic converges to 1 as the signature size converges to the total number of variables. Therefore, $k$ was chosen as a trade-off between signature size and stability, i.e. a signature size exhibiting maximal possible stability and being smaller than the total number of variables.

\section{Model building}

As multivariate survival models using microarray data are prone to overfitting, we built the model by combining the univariate Cox models computed during feature selection [8]. Each univariate model is defined as $\beta$ pclust $_{j}$, also referred as risk score in the literature. We used the sum rule as this method outperforms more complex combination schemes [7]. We set all the weights to 1 and computed the combined risk score as $\sum_{j=1}^{k} \beta$ pclust ${ }_{j}$.

\section{Method evaluation}

To avoid over-optimistic estimation of prediction accuracy, a leave-one-out cross-validation (LOOCV) and M10FLODCV procedures were used. As LOOCV does not depend on the order of patients in the dataset, these results will be discussed in details.

\section{Independent validation data sets}

Four independent validation sets were used to assess the performance of the classifier. These demographics are shown in [Additional file 3].

\section{Guy's hospital dataset (GUYT2)}

This external validation set was kindly provided by the Guy's Hospital, London, United Kingdom, consisting of 77 patients diagnosed with early stage breast cancer and treated with adjuvant tamoxifen monotherapy. Samples were hybridized using Affymetrix U133PLUS2 Genechips $^{\mathrm{TM}}$ according to standard Affymetrix protocols. Gene expression values from the CEL were normalized by use of the standard quantile normalization method in RMA [10] and are available from GEO database, accession number GSE9195.

\section{Dataset of Ma et al. (Ma)}

This dataset consisted of 60 patients diagnosed at the Massachusetts General Hospital, Boston, United States of America, and who were treated with adjuvant tamoxifen monotherapy. The samples were hybridized on the Agilent microarray platform and have been previously described [11]. The raw data was obtained at the GEO database (accession number GSE1378).

\section{Dataset of Reid et al. (Reid)}

This external validation set was kindly provided by the Department of Experimental Oncology, Istituto Nazionale per lo Studio e la Cura dei Tumori, Milan, Italy, consisting of 113 patients who had received adjuvant tamoxifen monotherapy. Samples were hybridized on their local cDNA microarray platform. Part of this dataset has previously been published [12]. We were unaware of 
the clinical data at all times and the survival analyses were performed in Milan.

Dataset of Jansen et al. (Jansen)

This dataset consisted of 112 patients diagnosed at the Erasmus MC, Rotterdam, Netherlands and who were treated with tamoxifen in the metastatic setting as first line hormonal therapy. Tumour response previously described [13] included both complete and partial responses and progressive disease. Samples were hybridized on $18 \mathrm{~K}$ human cDNA arrays manufactured at the Central Microarray Facility at the Netherlands Cancer Institute, Rotterdam, Netherlands. The raw data was kindly supplied by our Rotterdam colleagues.

Mapping across microarray platforms was done using the "Cleanex" database [14] to retrieve corresponding gene symbols and Affymetrix probe sets.

\section{Statistical analysis}

Although the risk score can be used as a continuous variable, we divided the dataset into two prognostic groups to generate a high or low risk status as this allowed us to estimate hazard ratios and produce Kaplan Meier curves. For the purposes of this study, a binary classification was generated using a 70:30 cutoff that is, $70 \%$ of samples would be considered low risk (hence, the majority of these patients would still suitable for tamoxifen) and 30\% high risk of relapse on adjuvant tamoxifen monotherapy. This cutoff was an arbitrary figure chosen by the authors to balance the cost of tamoxifen vs. other more expensive endocrine agents against relapse risk. The results shown in this manuscript were from analyses using the 70:30 cutoff for tamoxifen-treated and the external validation GUYT2 datasets, though similar results were obtained with a 50:50 cutoff. However, the samples in the Ma and Reid datasets were chosen to be balanced for recurrences within 5 years and non-recurrences after 6 years (case control studies, a non-consecutive series). Therefore, a 50:50 cutoff was used to take into account for the balanced number of events. Performance of the classifier computed by LOOCV was assessed using Kaplan-Meier survival curves and log rank p-values. The overall performance of the classifier in the three adjuvant data sets was estimated using classical meta-analysis methods [15].

Hazard ratios (HRs) for the risk groups defined by the classifier were calculated using a Cox's regression stratified by clinical center to account for possible heterogeneity in patient selection or other potential confounders among the various centers. For each independent validation data set, the HR (with their 95\% confidence intervals [CI]) was displayed on a forest plot and tested for heterogeneity using a chi-square test [15]. HRs were then combined using the inverse variance-weighted method with fixed effect model [15] to compute an overall HR.

To establish if the model predicted response to treatment, a univariate logistic regression model was used with the risk score as explanatory variable. Significance was determined by the Wald test and a false discovery rate (FDR) < 0.05 .

Statistical analysis was performed using SPSS statistical software package version 13.0 and the R software package version 2.3 [16].

\section{Correlation with the grade gene expression index (GGI)}

The Spearman's correlation between the risk scores produced by the predictor and that produced by GGI, previously described in Sotiriou et al, 2006 [5], was calculated to assess the contribution of proliferation-related genes to the prognostic ability of the current predictor.

\section{Network and pathway analysis}

Analysis of gene interactions for each cluster of the final classifier was performed using Ingenuity Pathways Analysis (IPA) tools version 3.0 [17]. Affymetrix probe sets of each cluster were used as input to generate biological networks based on a curated list of molecular interactions in IPA. IPA then calculated a significance value for enrichment of the functional classes and canonical pathways generated for each of these networks. Only significant functions and pathways are shown.

\section{Results}

\section{Predictor development}

Gene expression profiles of the 255 patients in the training set were used to derive the predictor. A signature of 13 clusters was assessed to be highly stable [Additional file 4] and hence chosen for further predictor development. In terms of performance, the best predictor used 45 clusters but a signature size of 13 performed similarly and had the advantage of using fewer clusters [Additional file 51]. Figure 2 shows the frequency of selection of each cluster in the M10FOLDCV process. All of the 13 clusters incorporated in the final predictor were the most frequently selected during the training phase.

\section{Classifier performance on the training set}

A risk score derived from 13 clusters was developed to predict the patient's risk of developing distant metastases as high or low risk. The final model consisted of 13 clusters and 239 associated probe sets (181 genes, [Additional file $2 \mathrm{~b}])$. The hazard ratio [15] for the occurrence of distant metastases using LOOCV was 3.86 (95\% CI: 2.32-6.41) p $<0.0001$ using the classifier as a binary variable. Results using M10FOLDCV were slighter lower however the difference was small (HR: 3.28, 95\%CI: 2.66-3.84, p < 


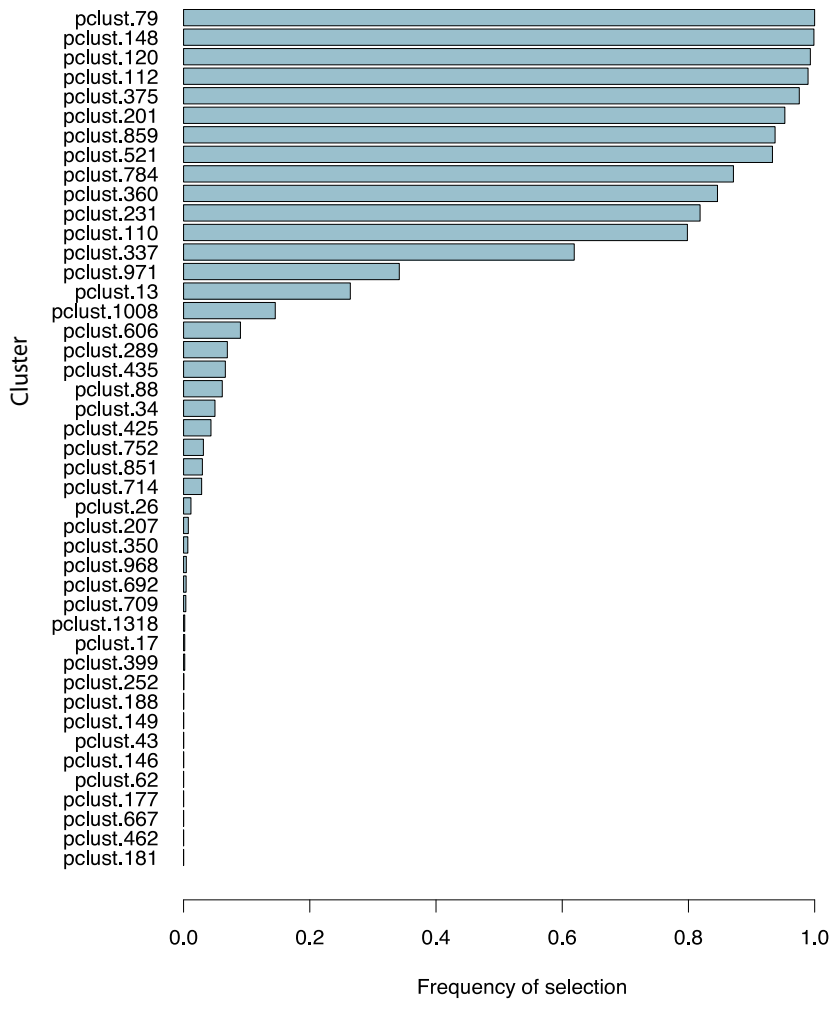

Figure 2

Signature stability. Signature stability demonstrating frequency of selection for the various clusters in multiple I0fold cross-validations.

0.0001). We further assessed the performance of our method by dividing the dataset into separate populations according to institution. The performance of the classifier algorithm on each population and corresponding 3-year DMFS is shown in Table 1, providing supporting evidence that the classifier algorithm is not population dependent. The survival curves estimated by Kaplan-Meier analysis are shown in Figure 3.

Univariate Cox regression analysis of the training set with the classifier and clinico-pathological prognostic factors known are shown in Table 2. The classifier gives the strongest $\mathrm{HR}$ with histological grade $(\mathrm{HR}=1.77,95 \% \mathrm{CI}$ : $1.17-2.68, \mathrm{p}=0.007)$ and tumor size $(\mathrm{HR}=2.18,95 \% \mathrm{CI}$ : $1.27-3,75, \mathrm{p}=0.003$ ) also significant. In the multivariate model, the classifier retained its significance as an independent variable for prediction of distant recurrence (HR $=3.26,95 \% \mathrm{CI}: 1.76-6.05, \mathrm{p}=0.0002$ ).

\section{Independent validation on external datasets: a meta- analysis}

Validation of the classifier was performed on three independent data sets (GUYT2, Ma, and Reid) consisting of

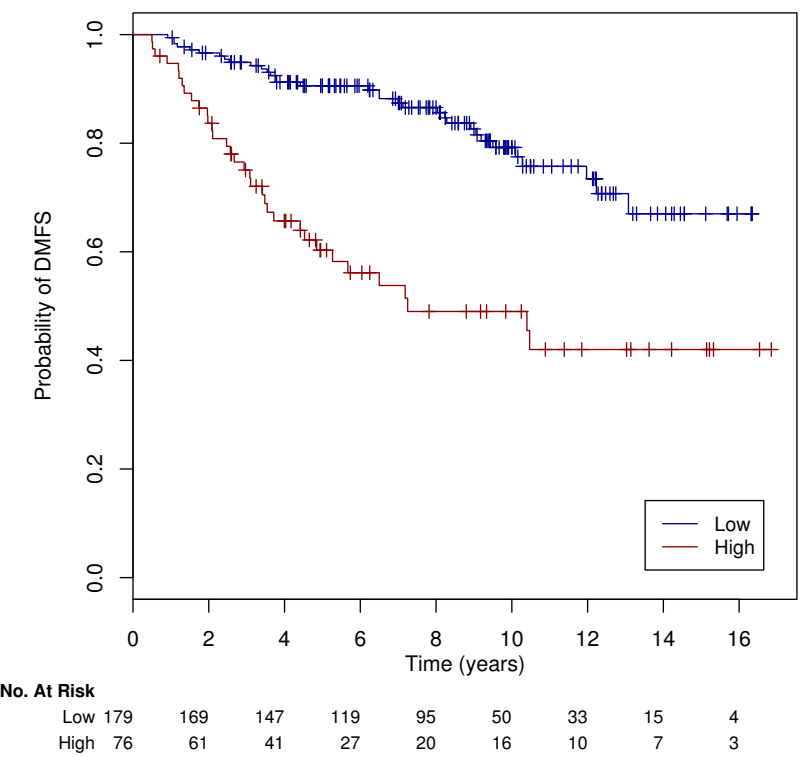

Figure 3

Survival curves for training set. Kaplan Meier curves for the binary classification computed using leave-one-out crossvalidation on the tamoxifen-treated dataset $(n=255)$. The two survival curves were significantly different according to the log rank test $(p<0.000 I)$.

250 samples taken from women at diagnosis and who had received the same adjuvant systemic therapy. These three datasets had been hybridized using different microarray platforms. The number of probe sets that could be mapped per cluster is shown in Table 3. Whilst the GUYT2 dataset was obtained from a consecutive series of patients (results by Kaplan Meier analysis shown in Figure 4a), the samples in the Ma and Reid datasets were chosen to be balanced for recurrences within 5 years and non-recurrences after 6 years (case control studies, results from Kaplan-Meier analysis shown in [Additional file 6]. The overall performance of the classifier in the 3 datasets is shown in Figure 4b. In the 250 women treated with adjuvant tamoxifen, the classifier was able to define two distinct prognostic groups (HR 2.01, 95\%CI: 1.29-3.13, p = $0.002)$. Interestingly, in the Affymetrix validation dataset (GUYT2), where all probe sets could be mapped, the performance of the classifier was the highest (Figure 4a: HR 4.02, 95\%CI: $1.13-14.27, \mathrm{p}=0.03$ ), suggesting that the validation may have been limited by technical factors. A multivariate analysis comparing other prognostic factors with the gene classifier was not performed due to a large number of missing values. However overall, this metaanalysis still provides evidence that our classifier has significant clinical value for prediction of distant relapses in patients treated with adjuvant tamoxifen monotherapy. 
Table I: Performance of the classifier. Performance of the 13 clusters classifier algorithm re-training and validation on the separate institutional populations using both leave-one-out and multiple 10 -fold cross-validations.

\begin{tabular}{|c|c|c|c|c|c|c|}
\hline \multirow[t]{2}{*}{$\begin{array}{c}\text { Training set (total/ } \\
\text { events) }\end{array}$} & \multirow[t]{2}{*}{$\begin{array}{c}\text { Validation set (total/ } \\
\text { events) }\end{array}$} & \multirow[t]{2}{*}{$\begin{array}{l}\text { Hazard ratio } \\
(95 \% \mathrm{Cl})\end{array}$} & \multirow[t]{2}{*}{$\begin{array}{l}\text { Log rank } p \\
\text { value }\end{array}$} & \multicolumn{3}{|c|}{$\begin{array}{c}\text { Distant Metastases Free Survival for low risk } \\
\text { group only\# }\end{array}$} \\
\hline & & & & DMFS at 3 years & DMFS at 5 years & DMFS at 10 years \\
\hline OXFT (99/19) & KIT/GUYT(I56/48) & $2.17(1.2-3.91)$ & $<0.00001$ & $91 \%$ & $87 \%$ & $79 \%$ \\
\hline KIT $(69 / 20)$ & OXFT/GUYT (I86/47) & $4.07(2.23-7.4 I)$ & $<0.00001$ & $96 \%$ & $92 \%$ & $88 \%$ \\
\hline GUYT (87/28) & OXFT/KIT (I68/39) & $5.93(3.0-11.75)$ & $<0.00001$ & $93 \%$ & $89 \%$ & $82 \%$ \\
\hline KIT/GUYT (156/48) & OXFT (99/19) & $14.59(5.38-39.5)$ & $<0.00001$ & $97 \%$ & $94 \%$ & $91 \%$ \\
\hline OXFT/GUYT (I86/47) & $\operatorname{KIT}(69 / 20)$ & $3.44(1.36-8.67)$ & 0.005 & $96 \%$ & $92 \%$ & $84 \%$ \\
\hline OXFT/KIT(I68/39) & GUYT (87/28) & $2.23(1.05-4.7 I)$ & 0.03 & $96 \%$ & $92 \%$ & $84 \%$ \\
\hline \multicolumn{2}{|c|}{ Leave-one-out cross validation (255/67)* } & $3.86(2.32-6.4 I)$ & $<0.0001$ & $94 \%$ & $91 \%$ & $84 \%$ \\
\hline \multicolumn{2}{|c|}{ Multiple I0-fold cross-validation (255/67) } & $3.23(2.66-3.84)$ & $<0.0001$ & $94 \%$ & $90 \%$ & $83 \%$ \\
\hline
\end{tabular}

\# as estimated by Kaplan Meier survival curves.

Patients samples obtained from: OXFT: John Radcliffe Hospital, Oxford, UK; KIT Uppsala University hospital, Uppsala, Sweden; GUYT Guys hospital, London, UK.

- Reported results.

\section{Prediction of response in metastatic breast cancer patients treated with tamoxifen}

In order to delineate whether our classifier was predicting response to tamoxifen and/or the intrinsic aggressiveness of a breast tumor (prognostic), we applied our classifier to a data set of women who had received tamoxifen in the advanced setting where response to the treatment was clearly defined [13]. Twenty-nine of the 79 probes that could be mapped were significantly associated with clinical response (complete or partial vs. progressive disease, false discovery rate $($ FDR $)<0.05)$ and 4 cluster groups seemed to have some predictive ability (FDR $<0.15$, pclusts 148,120,375,201). However, overall, we found that our classifier had no discrimination ability in this group of patients. Interestingly one cluster centroid, cluster 375, was significantly associated with response (FDR = 0.008 ), suggesting that this cluster of 3 genes [see Addi- tional file $2 \mathrm{~b}$ ] could predict response to tamoxifen treatment. These results could imply that our classifier is mainly prognostic, though as only $30.5 \%$ of probe sets were able to be mapped from the cDNA platform, technical limitations could have significantly contributed to these results.

\section{Correlation with the grade gene expression index (GGI)}

The GGI is an algorithm which can quantify the expression of proliferation genes in a breast tumor [5]. Given that many current prognostic predictors derive a significant proportion of their discriminatory ability from proliferation-related genes [5], we were interested to assess this in our current predictor. Despite the different discovery methods, the groupings produced by our classifier and the GGI were highly correlated: (GUYT2 0.91; Reid 0.86; Ma 0.69; Jansen 0.55; all p-values <0.05), suggesting a sig-

Table 2: Cox regression analysis. Univariate and multivariate Cox regression analysis for time to distant metastases in 255 patients.

\begin{tabular}{|c|c|c|c|c|}
\hline & \multicolumn{2}{|c|}{ Univariate analysis } & \multicolumn{2}{|c|}{ Multivariate analysis\# } \\
\hline & Hazard ratio $(95 \% \mathrm{Cl})$ & $\mathrm{P}$ & Hazard ratio $(95 \% \mathrm{Cl})$ & $P$ \\
\hline Histological grade (I vs. 2 vs. 3 ) & $3.14(1.37-7.17)$ & 0.007 & $0.94(0.36-2.42)$ & 0.9 \\
\hline Tumor size $(\leq 20 \mathrm{~mm}$ vs. $\geq 20 \mathrm{~mm})$ & $2.18(1.27-3.75)$ & 0.005 & $1.58(0.83-3)$ & 0.2 \\
\hline Nodal status (positive vs. negative) & $1.62(0.95-2.79)$ & 0.08 & $1.30(0.7 \mid-2.37)$ & 0.4 \\
\hline ER high vs. low expression & $0.86(1.18-0.522)$ & 0.5 & $0.97(0.56-1.7)$ & 0.9 \\
\hline PgR high vs. low expression & $0.42(0.25-0.7)$ & 0.0007 & $0.49(0.26-0.9)$ & 0.02 \\
\hline HER2 high vs. low expression & $0.88(0.55-1.42)$ & 0.6 & $0.66(0.37-1.18)$ & 0.2 \\
\hline 13 cluster gene classifier* & $3.86(2.32-6.4 I)$ & $<0.0001$ & $3.26(1.76-6.05)$ & 0.0002 \\
\hline
\end{tabular}

\#Multivariate model contained included 210 patients due to missing values, stratified by population.

*Binary classification using leave-one-out cross-validation.

**Age was not included in the model as $92 \%$ of patients were $\geq 50$ years of age.

ER: estrogen receptor status represented by ESRI Affymetrix probe set 205225_at.

PgR: progesterone receptor status represented by PGR Affymetrix probe set 208305_at.

HER2: represented by ERBB2 Affymetrix probe set 216836_s_at.

For ER, PgR and HER2, high vs. low expression groups was defined by generating groups at the median value. 
A

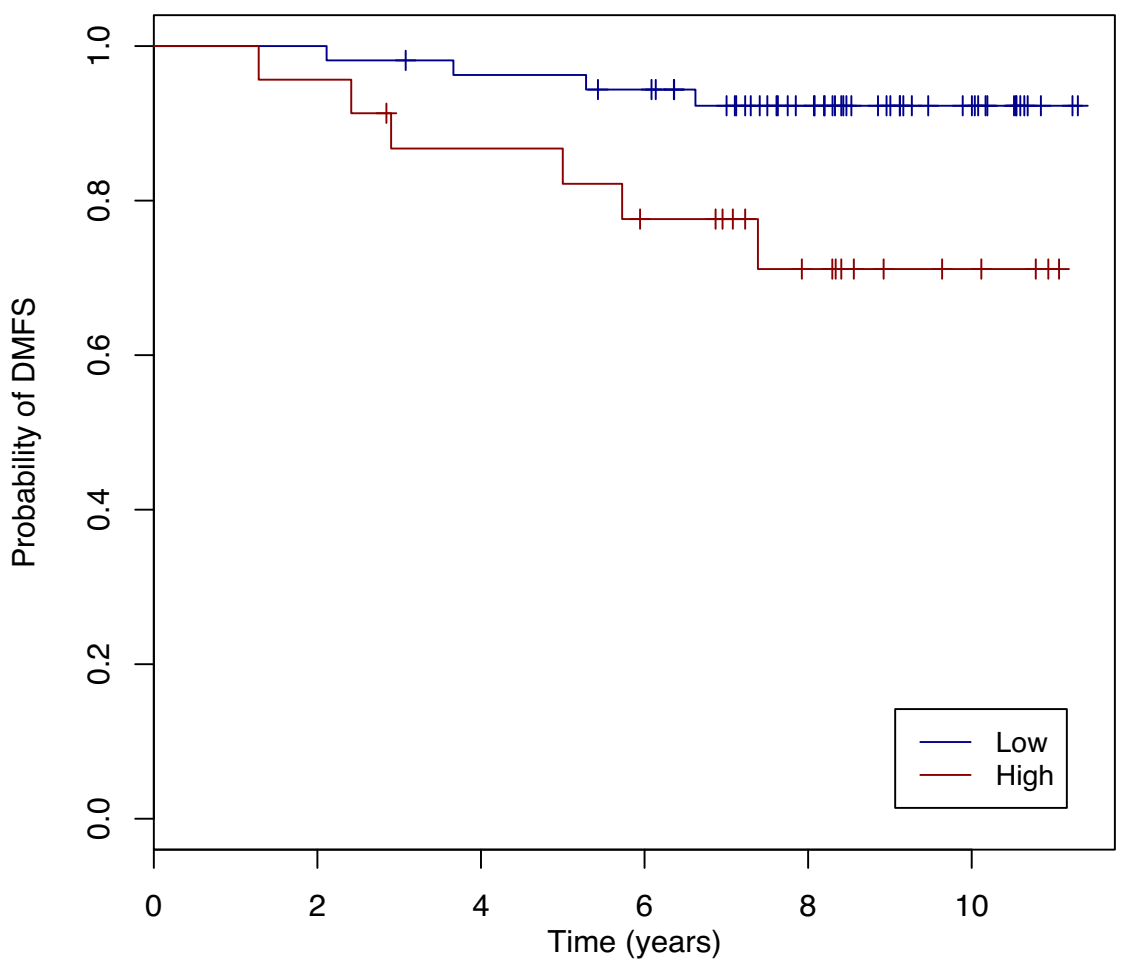

No. At Risk

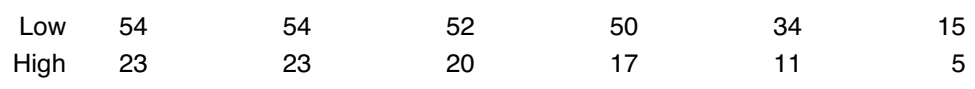

$\mathrm{B}$

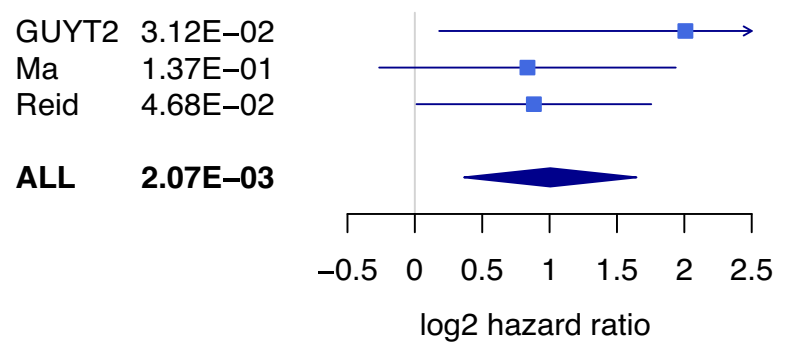

\begin{tabular}{|l|l|l|l|}
\hline Data set & Hazard Ratio & $95 \% \mathrm{Cl}$ & P value \\
\hline GUYT2 & 4.02 & $(1.13-14.27)$ & 0.03 \\
\hline Ma & 1.78 & $(0.83-3.83)$ & 0.1 \\
\hline Reid & 1.84 & $(1.01-3.37)$ & 0.05 \\
\hline ALL & 2.01 & $(1.29-3.13)$ & 0.002 \\
\hline Test for heterogeneity $\mathrm{p}=0.5$ & \\
\hline
\end{tabular}

\section{Figure 4}

External validation of the classifier. (a) Kaplan Meier curves for the GUYT2 dataset. The two survival curves were significantly different according to the log rank test $(p=0.03)$. (b) Forest plots of hazard ratios obtained from the three independent validation datasets. 
Table 3: Mapping. Number of probe sets able to be mapped across datasets during independent validations.

\begin{tabular}{|c|c|c|c|c|c|}
\hline \multirow[t]{2}{*}{ Cluster no. } & \multirow[t]{2}{*}{ Total probe sets present in classifier } & \multicolumn{4}{|c|}{ Mapped } \\
\hline & & GUYT2 dataset & Ma dataset & Reid dataset & Jansen dataset \\
\hline 79 & 7 & 7 & 3 & I & 2 \\
\hline 148 & 45 & 45 & 23 & 23 & 15 \\
\hline 112 & 14 & 14 & 5 & 4 & 9 \\
\hline 120 & 38 & 38 & 18 & 15 & 16 \\
\hline 375 & 8 & 8 & 4 & 3 & 3 \\
\hline 201 & 17 & 17 & 5 & 8 & 6 \\
\hline 521 & 19 & 19 & 10 & 8 & 5 \\
\hline 784 & 7 & 7 & 1 & I & 1 \\
\hline 859 & 7 & 7 & 4 & 2 & 2 \\
\hline 360 & 14 & 14 & 4 & 2 & 0 \\
\hline 231 & 26 & 26 & 8 & 4 & 9 \\
\hline 110 & 30 & 30 & 13 & 3 & 10 \\
\hline 337 & 7 & 7 & 4 & I & 1 \\
\hline Total n (\%) & $239(100 \%)$ & $239(100 \%)$ & 102 (42.6\%) & 75 (31.4\%) & 73 (30.5\%) \\
\hline
\end{tabular}

nificant proportion of its predictive power can be attributed to cell cycle-related genes.

\section{Functional analysis}

The biological functions of each of the 13 clusters were analyzed in the context of a curated list of published molecular interactions by IPA. Table 4 lists the high level functions and associated canonical pathways with statistically significant enrichment for each cluster. As seen, there are a number of gene clusters related to cell cycle function, supporting our finding above. Cluster 110 contains genes that have previously been associated with chemotaxis and invasion of breast cancer cell lines (SLIT2, RECK) $[18,19]$, as well as genes related to the extracellular matrix (ECM2, COL4A1). Less well characterized is the role of lipid metabolism (cluster 79) and immunological aspects in the differential response to tamoxifen (clusters 784, 375) though TNF alpha and TGF beta have previously been implicated in breast cancer development and progression [20]. Cluster 375, though small, is of interest, given its performance in the Jansen dataset. Functional analysis suggests that these genes (TGFBR4, PTGER4, C3, GNG2) are mainly involved in cellular inflammatory response and could be particularly important in determining the

Table 4: Functional analysis. Functional analysis of the 13 clusters from the gene signature (for full gene list [see Additional file $2 \mathrm{~b}$ ]).

\begin{tabular}{|c|c|c|c|c|}
\hline Cluster no. & Top Network overall & Top high level function & Top canonical pathway & $\begin{array}{c}\text { Number of focus genes able } \\
\text { to be mapped* }\end{array}$ \\
\hline 79 & $\begin{array}{l}\text { Cancer Inflammatory disease } \\
\text { Cell cycle }\end{array}$ & $\begin{array}{l}\text { Lipid metabolism Molecular } \\
\text { transport }\end{array}$ & cAMP mediated signaling & 3 \\
\hline 148 & Cancer Immune response & Cell cycle & I carbon pool by folate & 28 \\
\hline 112 & Gene expression & $\begin{array}{c}\text { Gene expression Protein } \\
\text { synthesis }\end{array}$ & EGF signaling & 10 \\
\hline 120 & Cell cycle Cellular movement & Cell cycle & G2/M checkpoint & 28 \\
\hline 375 & $\begin{array}{l}\text { Cellular movement, } \\
\text { inflammatory disease }\end{array}$ & Carbohydrate metabolism & TGF-beta signaling & 4 \\
\hline 201 & DNA recombination and repair & Cell cycle & GI/S checkpoint & 11 \\
\hline 521 & Cell cycle & Cell cycle & GI/S checkpoint & 14 \\
\hline 784 & Cell death & $\begin{array}{c}\text { Cell morphology Cellular } \\
\text { development }\end{array}$ & IL4 signaling & 3 \\
\hline 859 & Gene expression & Cell morphology & None given & 4 \\
\hline 360 & DNA recombination and repair & Cell cycle & None given & 6 \\
\hline 231 & $\begin{array}{l}\text { Cell to cell signaling and } \\
\text { interaction }\end{array}$ & Embryonic development & IGFI pathway & 13 \\
\hline 110 & Cell death Cellular development & Cancer Inflammation & PDGF signaling & 14 \\
\hline 337 & Cell morphology & $\begin{array}{l}\text { Cellular function and } \\
\text { maintenance }\end{array}$ & None given & I \\
\hline
\end{tabular}

* note that clusters (pclust) often contained probe sets that represented the same gene 
host's response to tamoxifen. The presence of gene cluster in our predictor that allude to other biological pathways apart from cell cycle function may facilitate further understanding of the upstream mechanisms behind tamoxifen resistance.

\section{Discussion}

Developing gene signatures that are stable, are effective at distinguishing prognostic groups and provide important biological information from whole genome microarray data remains a significant challenge. We propose a method which has similarities to a technique proposed by Bair and colleagues $[21,22]$, in combination with an estimation of signature stability [9] and to our knowledge, the largest dataset of ER+ patients homogenously treated in an attempt to address these issues. Whilst Bair et al. [21] used the clinical data to define a subset of survival-related genes prior to clustering, we performed an initial unsupervised clustering procedure to form the clusters which could act as biological networks, which were then used as single variables to build the classifier. We hypothesized that this would limit the effect the training set has over the final selection of genes for inclusion in the classifier [4] and allow a larger gene list for biological hypothesis generating. The inclusion of an assessment of "stability" facilitates determination of the most robust variables and hence presumably important biological information.

With this method, we were able to develop and validate a gene classifier that could predict which patients with ER+ $\mathrm{BC}$ were at high risk of relapse despite tamoxifen treatment. Importantly, we were able to validate the classifier on independent samples utilizing raw data from different microarray platforms using a meta-analytical approach. Demonstration of prognostic ability is important if we are to assemble gene lists from microarray data for biological hypotheses generation and potential laboratory experimental validation, which was one of the most important aims of this study. Validation of gene classifiers with independent samples from which they were developed from is a major challenge for microarray studies, especially those with clinical implications, and combining multiple datasets can be difficult due to different patient populations, sample preparation and microarray platforms. Our study uses one of the largest training and validation sets reported in the literature on tamoxifen (only) treated patients.

Whilst, in the future we may have a microarray-based diagnostic test incorporating all 181 genes in the 13 clusters, at present the routine use of this technology is not logistically feasible. However, the advantage of our approach is that as each cluster consists of a group of genes that are highly correlated and hence effectively act as one covariate. Thus, a diagnostic test of just 13 genes (one per cluster) could be developed for clinical use if desired, even though for biological study the researcher would be more interested in all the genes per cluster. To demonstrate this, we took a series of 13 individual probe sets (one per cluster) and correlated their performance with the full classifier on the training set of 255 patients. The median correlation was 0.94 (range: 0.88-0.97). The top 26 ranked 13-gene classifiers (with a correlation ranging from 0.95-0.97) and their corresponding probe sets are listed in [Additional file 7]. These "simple" tests will require further independent assessment but could be validated using immunohistochemistry or quantitative RTPCR and are attractive option for potential clinical implementation.

Due to the pressing clinical need, several other investigators have also developed gene predictors that can predict outcome in ER+ BC treated with adjuvant tamoxifen monotherapy $[11,13,23,24]$. These studies have used a variety of bioinformatics approaches to develop these gene signatures. These range from a candidate gene approach [24], selection of genes using a biological approach [23] and similar to our study, a discovery-based approach using supervised analyses correlated with clinical outcome [13]. Likewise, different patient populations were used in the development process. Ours is the only study to use a large, consecutive series of patients as a training set as opposed to samples obtained from a clinical trial [24]; or a case control population [11]. Only one of these reported gene classifiers has undergone noteworthy clinical validation [24], however unfortunately these genes provide no new potential therapeutic targets or insights into the underlying biology. Of note, we have previously published that proliferation-related genes are the common biological thread linking many of these currently published classifiers $[5,6]$. Our current classifier also has a significant amount of cell cycle genes, and is highly correlated with the GGI, but one of the aims of this study was to identify other potential biological mechanisms upstream of proliferation. All the clusters in the final classifier were the most common chosen during the cross-validation process suggesting the presence of other strong biological signals. Further experimental validation in in-vitro and in-vivo models will be required to test these hypotheses and their relevance to the clinical question. Interestingly, the cluster 375 was significantly predictive in the dataset of metastatic breast cancer patients treated with tamoxifen as first line treatment for relapsed disease. However, we were not able to validate the full gene classifier. The best approach on distinguishing prognosis versus therapy prediction using gene expression profiling remains unclear. It is possible that developing a predictor of true response to therapy may only be possible using samples from a randomized trial in the metastatic setting where response 
can be clearly defined and transcriptional profiles can be compared with an untreated control group.

\section{Conclusion}

Using a discovery-based whole genome approach, we have developed and validated a gene classifier that can distinguish patients at high risk of distant metastasis despite adjuvant tamoxifen monotherapy. In the future, these poor prognosis patients could be selected for prescription of other treatment modalities, such as chemotherapy and/or biological agents. In this study we propose an approach which has the advantage of facilitating both signature stability and biological interpretation. These are critical issues in the challenging task of building gene predictors for breast cancer patients as we endeavor to delineate meaningful biological and clinically useful information from the microarray-produced data.

\section{Authors' contributions}

$\mathrm{SL}, \mathrm{BH}-\mathrm{K}$ was responsible for the design and execution of the study, collation of study materials, the microarray analysis of study samples, the collection, assembly and verification of the data, data and statistical analysis and interpretation and final manuscript writing; CD assisted with the collation of study materials, the microarray analysis of study samples, data analysis and interpretation and final manuscript writing; PW assisted with the microarray analysis of study samples, data analysis and interpretation; FL provided technical support with the microarray analysis; AMT, PE, CG, KR, supplied the tissue samples from Guy's Hospital; JR, MGD, MAP performed the bioinformatics analysis using the Reid dataset in Milan; EMJJB, MPHMJ, JAF provided the microarray raw data for the Jansen dataset, and assisted with the data analysis and interpretation; MJP provided the study funding; MD, GB, and CS supervised the study. CS conceived the idea for the study. All authors read and approved the final manuscript.

\section{Additional material}

\section{Additional file 1}

Training dataset characteristics. Summary of patient and tumor characteristics of the 255 patients used in the development of the classifier. Click here for file

[http://www.biomedcentral.com/content/supplementary/1471-

2164-9-239-S1.xls]

\section{Additional file 2}

Probe clusters. (a) Full annotated gene list of all clusters (probe clusters; "pclust") used for classifier development after preliminary clustering and filtering on the separate dataset of 137 samples. (b) Full annotated gene list of probe clusters (pclust) used in final classifier and global model. Click here for file

[http://www.biomedcentral.com/content/supplementary/14712164-9-239-S2.xls]

\section{Additional file 3}

Independent validation datasets characteristics. Summary of patient and tumor characteristics of the four independent validation sets.

Click here for file

[http://www.biomedcentral.com/content/supplementary/1471-

2164-9-239-S3.xls]

\section{Additional file 4}

Signature size stability. Evolution of Stab criterion with respect to the signature size using multiple 10-fold cross-validation. The vertical dashed line represents the stability of the ranking using 13 pclusts. Click here for file

[http://www.biomedcentral.com/content/supplementary/14712164-9-239-S4.pdf]

\section{Additional file 5}

Signature size performance. Evolution of the $\log 2$ hazard ratio with respect to the signature size using multiple 10-fold cross-validation. The vertical dashed line represents the performance of the classifier using 13 pclusts. The horizontal red dashed line represents the limit of statistical significance.

Click here for file

[http://www.biomedcentral.com/content/supplementary/1471-

2164-9-239-S5.pdf]

\section{Additional file 6}

External validation of the classifier (Ma, Reid and Jansen datasets). (a) Kaplan Meier curves for Ma et al. The risk group was defined by the classifier using a 50:50 cutoff. The two survival curves were not significantly different according to the logrank test ( $p$-value of 0.1). (b) Kaplan Meier curves for Reid et al. The risk group was defined by the classifier using a 50:50 cutoff. The two survival curves were significantly different according to the logrank test ( $p$-value of 0.05). (c) Kaplan Meier curves for Jansen et al. The risk group was defined by the classifier using a 50:50 cutoff. The two survival curves were significantly different according to the logrank test (p-value of 0.25).

Click here for file

[http://www.biomedcentral.com/content/supplementary/14712164-9-239-S6.pdf]

\section{Additional file 7}

Simplified classifiers. Top correlated "simplified" classifiers of 13 genes (one probe set per cluster) and histogram displaying correlation between "simple" classifiers and final model.

Click here for file

[http://www.biomedcentral.com/content/supplementary/1471-

2164-9-239-S7.xls]

\section{Acknowledgements}

Sherene Loi is supported by the American Society of Clinical Oncology (ASCO) Young Investigators' grant and the National Breast Cancer Foundation of Australia. Christos Sotiriou, Christine Desmedt and Benjamin Haibe-Kains are supported by the Belgian National Foundation for Cancer Research. Christos Sotiriou is supported by the E. Lauder Breast Cancer Foundation, and the MEDIC Foundation.

\section{References}

I. Sorlie T, Perou CM, Tibshirani R, Aas T, Geisler S, Johnsen H, Hastie $\mathrm{T}$, Eisen MB, van de Rijn M, Jeffrey SS, Thorsen T, Quist H, Matese JC, Brown PO, Botstein D, Eystein Lonning P, Borresen-Dale AL: Gene 
expression patterns of breast carcinomas distinguish tumor subclasses with clinical implications. Proc Natl Acad Sci U S A 200I, 98(19): I0869-10874.

2. Sotiriou C, Neo SY, McShane LM, Korn EL, Long PM, Jazaeri A, Martiat P, Fox SB, Harris AL, Liu ET: Breast cancer classification and prognosis based on gene expression profiles from a population-based study. Proc Natl Acad Sci U S A 2003, I00(18): 10393-10398

3. Michiels S, Koscielny S, Hill C: Prediction of cancer outcome with microarrays: a multiple random validation strategy. Lancet 2005, 365(9458):488-492.

4. Ein-Dor L, Kela I, Getz G, Givol D, Domany E: Outcome signature genes in breast cancer: is there a unique set? Bioinformatics 2005, 2 I(2): I7I- 178.

5. Sotiriou C, Wirapati P, Loi S, Harris A, Fox S, Smeds J, Nordgren H, Farmer P, Praz V, Haibe-Kains B, Desmedt C, Larsimont D, Cardoso F, Peterse H, Nuyten D, Buyse M, Van de Vijver MJ, Bergh J, Piccart $M$, Delorenzi M: Gene expression profiling in breast cancer: understanding the molecular basis of histologic grade to improve prognosis. J Natl Cancer Inst 2006, 98(4):262-272.

6. Loi S, Haibe-Kains B, Desmedt C, Lallemand F, Tutt AM, Gillet C, Ellis P, Harris A, Bergh J, Foekens JA, Klijn JG, Larsimont D, Buyse M, Bontempi G, Delorenzi M, Piccart MJ, Sotiriou C: Definition of clinically distinct molecular subtypes in estrogen receptorpositive breast carcinomas through genomic grade. I Clin Oncol 2007, 25(1 0): | 239-1246.

7. Kittler J, Hatef M, Duin R, Matas J: On Combining Classifiers. IEEE Transactions on Pattern Analysis and Machine Intelligence 1998 10(3):226-238.

8. Haibe-Kains B, Desmedt C, Loi S, Delorenzi M, Sotiriou C, Bontempi G: Computational Intelligence in Clinical Oncology- a case study. In Studies in Computational Intelligence Volume Applications of computational intelligence in bioinformatics and biomedicine:current trends and open problems. Edited by: Smolinski TG, Milanova MM, Hassanien AE. Springer-Verlag; 2008.

9. Davis CA, Gerick F, Hintermair V, Friedel CC, Fundel K, Kuffner R, Zimmer R: Reliable gene signatures for microarray classification: assessment of stability and performance. Bioinformatics 2006, 22(19):2356-2363.

10. Bolstad BM, Irizarry RA, Astrand M, Speed TP: A comparison of normalization methods for high density oligonucleotide array data based on variance and bias. Bioinformatics 2003, I 9(2): $185-193$.

II. Ma XJ, Wang Z, Ryan PD, Isakoff SJ, Barmettler A, Fuller A, Muir B, Mohapatra G, Salunga R, Tuggle JT, Tran Y, Tran D, Tassin A, Amon P, Wang W, Enright E, Stecker K, Estepa-Sabal E, Smith B, Younger J, Balis U, Michaelson J, Bhan A, Habin K, Baer TM, Brugge J, Haber DA, Erlander MG, Sgroi DC: A two-gene expression ratio predicts clinical outcome in breast cancer patients treated with tamoxifen. Cancer Cell 2004, 5(6):607-6I6.

12. Reid JF, Lusa L, De Cecco L, Coradini D, Veneroni S, Daidone MG Gariboldi M, Pierotti MA: Limits of predictive models using microarray data for breast cancer clinical treatment outcome. J Natl Cancer Inst 2005, 97( I 2):927-930.

13. Jansen MP, Foekens JA, van Staveren IL, Dirkzwager-Kiel MM, Ritstier K, Look MP, Meijer-van Gelder ME, Sieuwerts AM, Portengen $H$, Dorssers LC, Klijn JG, Berns EM: Molecular classification of tamoxifen-resistant breast carcinomas by gene expression profiling. J Clin Oncol 2005, 23(4):732-740.

14. Praz V, Jagannathan V, Bucher P: CleanEx: a database of heterogeneous gene expression data based on a consistent gene nomenclature. Nucleic Acids Res 2004, 32(Database issue):D542-7.

15. Cochrance WG: Problems arising in the analysis of a series of similar experiments. Journal of the Royal Statistical Society 1937, 4:102-118.

16. Team RCD: The R Project for Statistical Computing. [http:// www.r-project.org].

17. Systems I: Ingenuity Pathway Analysis. [http://www.ingenu ity.com].

18. Liu LT, Peng JP, Chang HC, Hung WC: RECK is a target of Epstein-Barr virus latent membrane protein I. Oncogene 2003, 22(5 I):8263-8270.

19. Prasad A, Fernandis AZ, Rao Y, Ganju RK: Slit protein-mediated inhibition of CXCR4-induced chemotactic and chemoinva- sive signaling pathways in breast cancer cells. J Biol Chem 2004, 279(10):9115-9124.

20. Turner S, J AS, Cameron D: Tamoxifen treatment failure in cancer and the nonlinear dynamics of TGFbeta. J Theor Biol 2004, 229(I): I0I-III.

2I. Bair E, Tibshirani R: Semi-supervised methods to predict patient survival from gene expression data. PLoS Biol 2004, 2(4): EI08.

22. Park MY, Hastie T, Tibshirani R: Averaged gene expressions for regression. Biostatistics 2007, 8(2):2/2-227.

23. Oh DS, Troester MA, Usary J, Hu Z, He X, Fan C, Wu J, Carey LA, Perou CM: Estrogen-regulated genes predict survival in hormone receptor-positive breast cancers. J Clin Oncol 2006, 24(II): 1656-1664.

24. Paik S, Shak S, Tang G, Kim C, Baker J, Cronin M, Baehner FL, Walker MG, Watson D, Park T, Hiller W, Fisher ER, Wickerham DL, Bryant J. Wolmark N: A multigene assay to predict recurrence of tamoxifen-treated, node-negative breast cancer. N Engl J Med 2004, 35 I (27):28I7-2826.
Publish with Biomed Central and every scientist can read your work free of charge

"BioMed Central will be the most significant development for disseminating the results of biomedical research in our lifetime. "

Sir Paul Nurse, Cancer Research UK

Your research papers will be:

- available free of charge to the entire biomedical community

- peer reviewed and published immediately upon acceptance

- cited in PubMed and archived on PubMed Central

- yours - you keep the copyright 